\title{
DYNAMICS OF PINNED CHARGE DENSITY WAVES: NUMERICAL SIMULATIONS
}

\author{
E. VEERMANS and A. ERZAN ${ }^{1}$ \\ Solid State Laboratory, University of Groningen, Melkweg 1, 9718 EP Groningen, \\ The Netherlands
}

\section{R. HEIJUNGS}

Laboratory of Physiology and Physiological Physics, University of Leiden, P.O. Box 9604, 2300 RC Leiden, The Netherlands

\section{PIETRONERO}

Dipartimento di Fisica, Università di Roma "La Sapienza", Ple. Aldo Moro 2, 00185 Roma, Italy

\section{Received 19 March 1990}

A classical many-body model for the phase dynamics of randomly pinned charge density waves is found to exhibit stretched exponential relaxation below threshold. The statistics of single particle relaxation is highly non-trivial and can only be deduced from known scaling behaviour of intermittent trajectories in an uncoupled approximation. This is compared with results from present single-particle approaches. The self-similarity properties at the dynamical phase transition at threshold are investigated.

\section{Introduction}

Charge density wave (CDW) systems have been found to exhibit a large number of interesting phenomena besides nonlinear conductivity $[1,2]$, such as nonexponential relaxation below a finite threshold field for the onset of conduction [3], as well as mode locking [4] and memory effects [5].

The treatment by Pietronero and Strässler $[6,7]$ of the equations of motion of the phase modes of the Fröhlich CDW pinned by random impurities, taking full account of the internal degrees of freedom, provides a microscopic model, whose dynamical properties may be investigated in detail. Numerical treatment of their model yields $[6,7]$ a finite threshold field above which no equilibrium solutions for the polarization exist. They have also numerically obtained the dependence of the threshold field on the elastic coupling strength, i.e., the

\footnotetext{
${ }^{1}$ Present address: ICTP, P.O. Box 586, 34100 Trieste, Italy.
} 
pinned-unpinned phase diagram, and investigated the narrow as well as the broad band noise exhibited by the current in the unpinned region. Simulations performed on the same system by Littlewood [8] shed light on the properties of metastable states, which lead to hysteresis and memory effects. The pulseduration memory effect exhibited by an analogous model has been investigated by Coppersmith and Littlewood [9] and Tang et al. [10].

The set of equations for the phase modes of the CDW are in the form of an array of coupled maps [11], which have recently become the subject of active research in that they are found to give rise to spatio-temporal organizing behaviour of great richness [12]. It has been noted [10] that such models share, together with spin glasses, neural networks and fully turbulent flows, the property that their dynamics cannot be adequately described by a few effective degrees of freedom.

In this paper we concentrate on the relaxation behaviour of the classical CDW model of Pietronero and Strässler [6] below the threshold. The numerical integration of the equations of motion for the phases at the impurity sites, or the iteration of the equivalent coupled map lattice (CML) [11] yields information about the individual trajectories as well as the macroscopic behaviour of such quantities as the polarization and the current. Thus we have been able to investigate separately the consequences of random pinning (configurational noise) and the high effective dimensionality $[9,10]$ of the CML. The qualitative differences found between the coupled and uncoupled cases may help to understand the shortcomings of single particle or mean field approaches better [13-15].

Our main finding for the randomly pinned CDW system [6,7] is that in the presence of an external field smaller than the threshold value, the polarization and the current relax to their equilibrium values with a stretched exponential law of the form $\exp \left(-t^{\beta}\right)$. We find this to be the case both for random initial conditions and initial conditions obtained by previous relaxation to equilibrium in zero external field. This is to be contrasted with the response obtained after equilibration at a finite field. Turning off the field then yields an exponential decay to the new equilibrium state. For any finite sample, regardless of initial conditions, the extreme long time decay is, of course, once more pure exponential. We have performed extensive simulations in order to determine the value of $\beta$, which is found to be $\beta \sim 1 / 3$ well below the threshold, crossing over to a higher value in a neighborhood of the threshold. We have also computed the power spectrum of the noise in the current and found it to be Gaussian below the threshold. Right at the threshold, one finds a $1 / f$ dependence.

We have also investigated the spatio-temporal organization arising in a cellular automaton representation of the underlying model, and determined the fractal dimension of the patterns obtained. 
In section 2 we present the array of coupled maps for the phase dynamics of a randomly pinned CDW and we give a detailed description of the microscopic relaxation process. Availing ourselves of the results on the scaling behaviour of one-dimensional maps displaying Pomeau-Manneville type intermittency [1618], we given an analytic derivation of the power law decay of the current for an ensemble of uncoupled maps with configurational disorder. In section 3 we present our numerical results. After general remarks on the computational procedures used, we report our findings on simulations for (i) ensembles of uncoupled, single particle maps with configurational and temporal noise, (ii) an array of coupled map lattice describing a randomly pinned CDW. The main results of this section are summarized and briefly discussed in section 3.1. In section 4 we present our findings on the spatio-temporal patterns associated with the relaxation phenomenon.

\section{Phase dynamics of the charge density wave}

\subsection{The model}

A one-dimensional metal that undergoes a Peierls instability will exhibit a periodic modulation of the charge density of the form [6,7]

$$
\rho(x)=e \rho_{0}\left\{1+C \cos \left[q_{0} x+2 \pi \psi(x)\right]\right\} .
$$

The one-dimensional electron density and Fermi wavevector are denoted by $e \rho_{0}$ and $q_{0}$, respectively. In a Peierls system with a constant density of states per site $N_{0}$, the amplitude of the charge density wave (CDW), $C$, is given by $C=N_{0} \Delta / a \lambda \rho_{0}[6,7]$, where $a$ is the lattice constant, $2 \Delta$ is the gap and $\lambda$ is the electron-phonon coupling. It is assumed that the amplitude modulations can be neglected and the phase $\psi(x)$ denotes the position of the CDW. It is only these excitations that carry an electric current [2].

The effective Hamiltonian for the phase dynamics is given by $[2,6,7]$

$$
H_{0}=\frac{1}{q_{0}^{2}} \int \mathrm{d} x\left[\frac{1}{2} \rho_{m}\left(\frac{\partial \psi}{\partial t}\right)^{2}+\frac{1}{2} K\left(\frac{\partial \psi}{\partial x}\right)^{2}\right]
$$

where $\rho_{m}$ is the effective mass density of the CDW and for a Peierls system the elastic force is $K=\rho_{0} m v_{\mathrm{F}}^{2}$, where $m$ is the electron mass and $v_{\mathrm{F}}$ the Fermi velocity.

Choosing delta-function pinning potentials at the random impurity positions $x_{j}$ and a damping force only acting at these sites, Pietronero and Strässler [6] have been able to obtain a difference equation for the values of the phase at 
the impurity sites,

$$
\frac{\mathrm{d} \psi_{j}}{\mathrm{~d} t}=B\left(\frac{\psi_{j+1}-\psi_{j}}{u_{j+1}-u_{j}}-\frac{\psi_{j}-\psi_{j-1}}{u_{j}-u_{j-1}}\right)-\sin \left[2 \pi\left(Q_{0} u_{j}+\psi_{j}\right)\right]+E Q_{j} .
$$

Here dimensionless parameters have been introduced such that $u_{j}=x_{j} \nu$, where $\nu$ is the one-dimensional impurity concentration, $Q_{0}=q_{0} / 2 \pi \nu, Q_{j}=\frac{1}{2}\left(u_{j+1}-\right.$ $\left.u_{j-1}\right)$ and the time has also been normalized. The effective coupling constant is $B=2 \pi K \nu / e \rho_{0} C V_{0} q_{0}^{3}$, and the electric field $E$ is normalized by $C V_{0} \nu q_{0}$.

Defining a random pinning phase $\varphi_{j} \equiv x_{j} q_{0} / 2 \pi$ and finally neglecting randomness in the spacing of the impurities in the elastic and external field terms, which only introduce effects of higher order, we obtain

$$
\frac{\partial \psi_{j}}{\partial t}=B\left(\psi_{j+1}-2 \psi_{j}+\psi_{j-1}\right)-\sin \left[2 \pi\left(\psi_{j}+\psi_{j}\right)\right]+E
$$

If we also discretize time $(t=n \mathrm{~d} t)$ we get a set of $N$ diffusively coupled maps which enables us to numerically describe the time evolution of $N$ coupled phases $\psi$ by iterating from some starting configuration. Thus, with

$$
\psi_{j}(n+1)=\psi_{j}(n)+\dot{\psi}_{j}(n) \mathrm{d} t
$$

we have

$$
\begin{aligned}
\psi_{j}(n+1)= & (1-2 B \mathrm{~d} t) \psi_{j}(n)+B \mathrm{~d} t\left[\psi_{j-1}(n)+\psi_{j+1}(n)\right] \\
& -\mathrm{d} t \sin \left[2 \pi\left(\psi_{j}(n)+\varphi_{j}\right)\right]+E \mathrm{~d} t
\end{aligned}
$$

We may define a polarization via

$$
P(t)=\frac{1}{N} \sum_{j}^{N} \psi_{j}(t)
$$

and a current density

$$
J(t)=\frac{\partial P(t)}{\partial t}=\frac{1}{N} \sum_{j}^{N} \dot{\psi}_{j}(t) .
$$

The simulations performed by Pietronero and Strässler [6, 7] on the system given by (2.3) have shown that there is a maximum value of the field $E_{\mathrm{th}}$, such that for $E>E_{\text {th }}$, there is no equilibrium solution for $P(t)$, thus $J(t)$ is a nondecaying function of time, while for fields below threshold the system tends to an equilibrium value of the polarization. In fig. 1 we reproduce their phase 


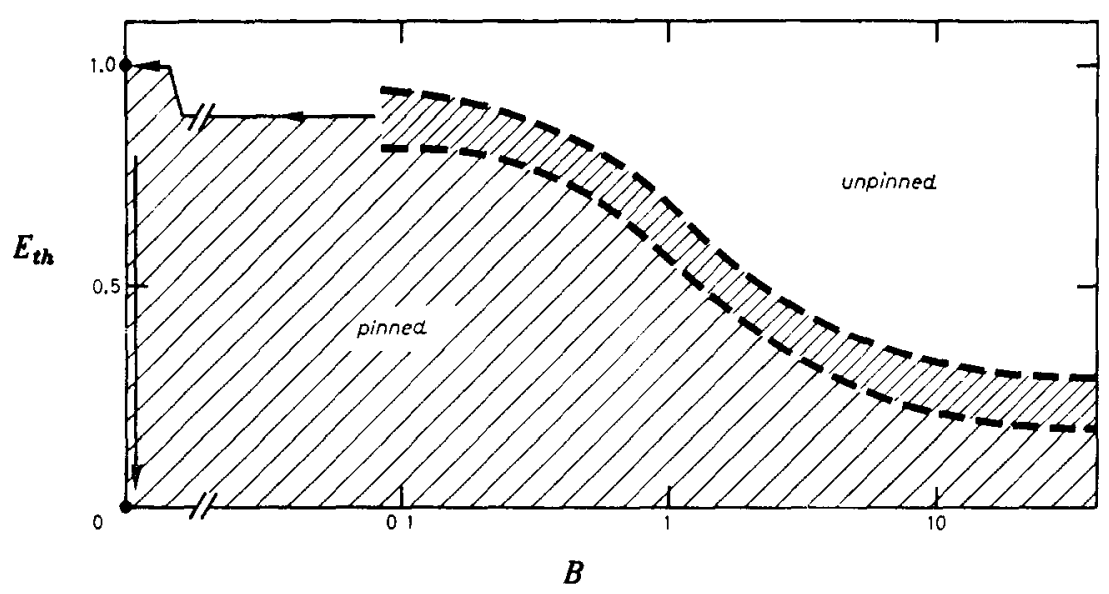

Fig. 1. Pinned verus unpinned phase diagram. We show here the dependence of the threshold field $E_{\mathrm{th}}$ on the coupling $B$. For $B \rightarrow 0$ the behaviour of $E_{\mathrm{th}}$ is non-analytic. Taken from Pietronero and Strässler [6], with permission of the authors.

diagram. The boundary between the pinned and unpinned regions is a broad band, attesting to the fact, which we verify, that the threshold field $E_{\text {th }}$ differs from sample to sample. Versteeg has also been able to obtain $E_{\mathrm{th}}$ analytically in a mean field type approach to the present model [19].

In fig. 2, we display a typical decay curve of the current for a chain of $N=10^{4}$ points. In (2.6), the values of $\varphi_{i}$ as well as the initial values of the $\psi_{i}$ have been chosen randomly within the interval $[0,1]$. The field has been set equal to 0.5 and $B=1$. For small $t$ the curve is relatively smooth, but as time passes it gets more and more irregular until, at very large times, one is able to distinguish isolated peaks of a characteristic shape. We have verified, by examining chains of various lengths, that the current in all cases is a superposi-

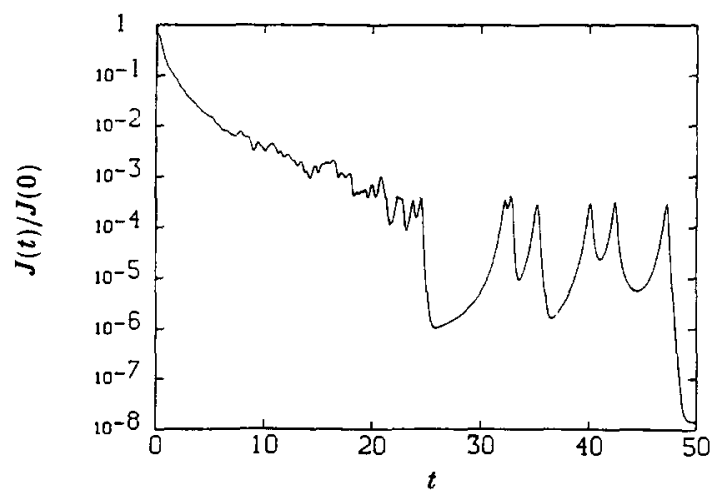

Fig. 2. Polarization current for a coupled map with random pinning $(E=0.5, B=1)$. 
tion of single events, or contributions from the relaxation of individual points on the chain. Any given point on the chain undergoes typically a single relaxation (unless the field is very close to threshold) and the distribution of these single relaxation events in time, or "waiting time distribution" is what determines the shape of the overall decay. In subsequent sections we will present numerical results on the waiting time distribution, which turns out in all cases to have a long-time tail. This is graphically illustrated by the fact that however large $N$ may be, the current has the characteristic shape shown in fig. 2 , with very long waiting times showing up as isolated peaks.

\subsection{The relaxation mechanism}

In order to clarify the origin of the individual relaxation events, we cast (2.6) into the form

$$
\psi_{j}(n+1)=f_{j}\left[\psi_{j}(n)\right]+B \mathrm{~d} t\left[\psi_{j-1}(n)+\psi_{j+1}(n)\right]=f_{j}\left[\psi_{j}(n)\right]+E_{\text {eff }} \mathrm{d} t
$$

with

$$
f_{j}[x]=(1-2 B \mathrm{~d} t) x-\mathrm{d} t \sin \left[2 \pi\left(x+\varphi_{j}\right)\right]+E \mathrm{~d} t .
$$

The first equation above is now in the form of a one-dimensional map with a time dependent force term. For the rest of this section we will concentrate on the one-dimensional map appearing in (2.10). Changing the notation slightly, let us denote by $x_{n}$ the $n$th iterate of the argument. Moreover we will drop the site index from the pinning phase $\varphi$. Recall from (2.6) that

$$
f\left(x_{n}\right)=x_{n}+\dot{x}\left(x_{n}\right) \mathrm{d} t,
$$

where

$$
\dot{x}\left(x_{n}\right)=-2 B x_{n}-\sin \left[2 \pi\left(x_{n}+\varphi\right)\right]+E,
$$

which gives

$$
x_{n+1}=f\left(x_{n}\right)=(1-2 B \mathrm{~d} t) x_{n}-\mathrm{d} t \sin \left[2 \pi\left(x_{n}+\varphi\right)\right]+E \mathrm{~d} t .
$$

In fig. 3 we plot this map for $E=0$ and $E=1$, with $\varphi=\frac{1}{2}$. It has fixed points when $x_{n+1}=x_{n}$, i.e. $\dot{x}\left(x_{n}\right)=0$, which are either repulsive or attractive depending on whether the absolute value of the derivative of (2.11) with respect to $x$ is bigger or smaller than unity. The map with $E=0$ has two attractive fixed 


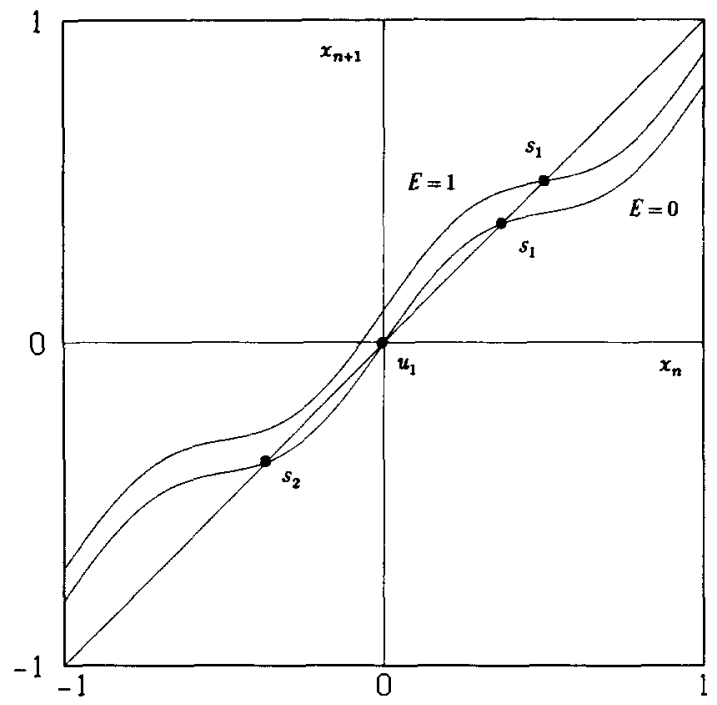

Fig. 3. One-particle map for $E=0(B=1, \varphi=5, \mathrm{~d} t=0.1)$. When a field $E=1$ is put on, a neck appears. Within this neck the iteration velocity is slow (laminar zone), afterwards fast (event).

points $s_{1}$ and $s_{2}$. A phase point or "particle" inserted before $s_{2}$ will, under iterations of this map, converge to $s_{2}$ and remain there. Had $E$ been sufficiently large, however, or with a sudden rise of the field, the fixed points at $s_{2}$ and the origin would collapse and annihilate, creating a narrow neck through which the particle will slowly iterate, as is the case with $E=1$. Once the narrow neck region is left behind, there is a sudden rise in the velocity with a well defined maximum and then the trajectory converges exponentially to the fixed point $s_{1}^{\prime}$.

We illustrate this in fig. $4 \mathrm{a}$, where we plotted $\dot{\psi}_{j}(t)$ for a number of sites belonging to a coupled system (2.9) of $N=500$ points. The corresponding $\psi_{j}(t)$ are shown in fig. $4 \mathrm{~b}$. One clearly notices the laminar zones $\left(\dot{\psi}_{j}(t)\right.$ small, $\psi_{j}(t)$ almost constant), the sudden bursts and the convergence to the fixed points. This well confined maximum in the velocity corresponds to the peaks seen in the current (recall that $J=\langle\dot{\psi}\rangle$ ) and is the signature of an individual relaxation event. Then one is able to associate the waiting time with the path length $[16,17]$, or the number of iterations it takes for an individual trajectory to leave behind a neck region.

The phenomena associated with a tangent bifurcation are well known within the context of the Pomeau-Manneville intermittent route to chaos [16]. Pomeau and Manneville [16] and Hirsch et al. [17] have determined the scaling behaviour of the path length $\tau$ in terms of $\varepsilon$, the width of the neck, once an "acceptance window" is chosen to delimit the neck region. They find that $\tau \sim \varepsilon^{-1 / 2}$ when the map has a quadratic extremum at the point of nearest 


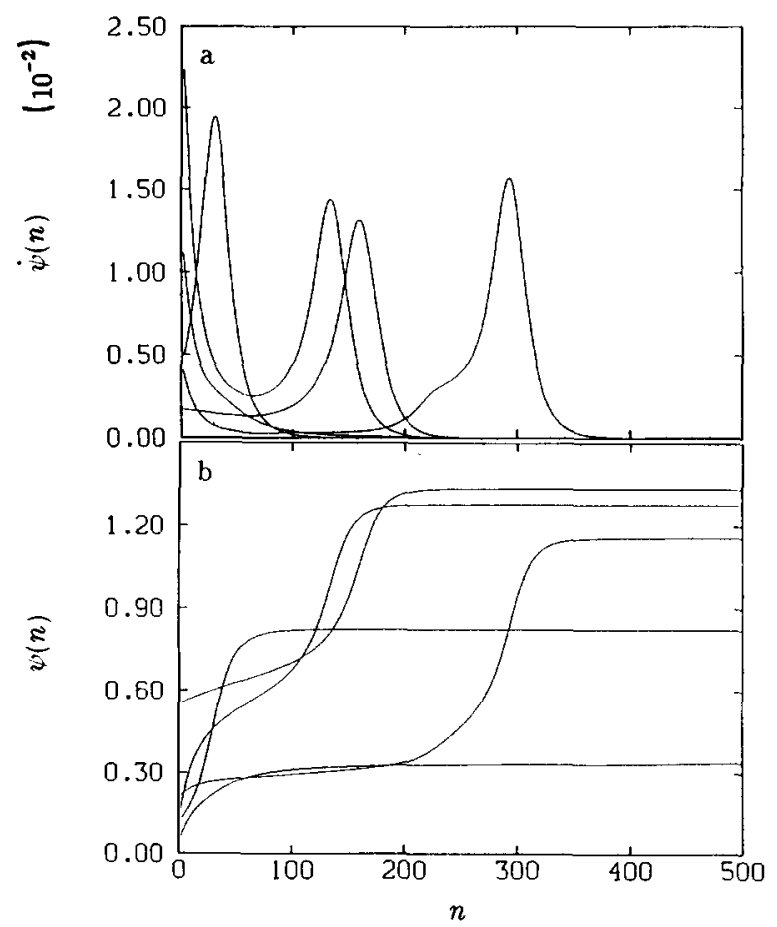

Fig. 4. (a) Velocity $\dot{\psi}$ plotted versus time for five sites of a coupled map with random pinning showing four events $(E=0.5, B=1, N=500, \mathrm{~d} t=0.01)$ with in (b) the corresponding $\psi$ 's.

approach to the diagonal. Eckmann et al. [18] have shown that this scaling behaviour survives in the presence of delta-correlated time-dependent noise.

With an event defined as the occurrence of a maximum in the velocity and the waiting time $\tau$ as the number of iterations before the manifestation of the event, we are able to compute numerically the waiting time distribution for the dynamics of a chain of $N$ phase variables. When both $\ddot{\psi}_{j}(n)$ changes sign and $\dot{\psi}_{j}(n)$ exceeds threshold $a$, the count in the bin with $\tau=n$ is increased by 1 . In fig. 5 we provide evidence that the current $J$ is nothing more than a smoothed version of the histogram $\mathcal{N}_{\tau}(\tau)$, or that

$$
J(t) \sim \int \mathcal{N}_{\tau}(\tau) \delta(t-\tau) \mathrm{d} \tau
$$

If we can describe the distribution of waiting times, we can describe $J$. It turns out, however, that this distribution can be derived analytically, from the microscopic equations of motion and known scaling behaviour, only in very restricted cases. 


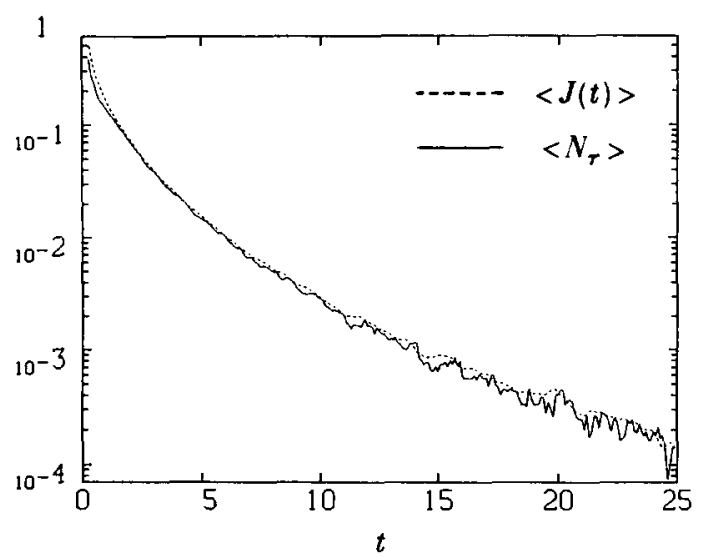

Fig. 5. Current $J$ and waiting time distribution $\mathcal{N}_{\tau}$ for a system of coupled maps with random pinning ( $E=0.5, B=1,50$ samples). $\mathcal{N}_{\tau}$ is normalized to $J$.

\subsection{Statistics of single particle relaxation}

In the next section we will present in detail our numercial results on $\mathcal{N}_{\tau}(\tau)$. In the remainder of this section we proceed to give a derivation of the power law dependence of $\mathcal{N}_{\tau}(\tau)$ when the chain is treated as an ensemble of one-dimensional maps (2.10), with a random distribution of $0<\varphi<1$. Physically this is equivalent to a single-particle approximation where the neighboring phases are assumed to remain fixed as a given $\psi_{j}$ undergoes a transition. It will become immediately clear from the computation below that in this single-particle approach spatial fluctuations in the external field, or, equivalently, the values of the neighboring phase points, enter on the same footing as the fluctuations in the pinning phase, and will give rise to the same type of anomalous relaxation behaviour.

Let us expand our map (2.10) around the point of near tangency $\left(x_{\text {neck }}\right)$,

$$
\begin{aligned}
f\left(x_{n}\right)= & f\left(x_{\text {neck }}\right)+\left.\frac{\partial f\left(x_{n}\right)}{\partial x_{n}}\right|_{x_{\text {neck }}}\left(x_{n}-x_{\text {neck }}\right)+\left.\frac{1}{2} \frac{\partial^{2} f\left(x_{n}\right)}{\partial x_{n}^{2}}\right|_{x_{\text {neck }}}\left(x_{n}-x_{\text {neck }}\right)^{2} \\
& +\mathscr{O}\left(x_{n}^{3}\right) .
\end{aligned}
$$

Noting that $\left.\left(\partial x_{n+1} / \partial x_{n}\right)\right|_{x_{\text {neck }}}=1$, one finds

$$
x_{n+1}=\varepsilon+x_{n}+\kappa\left(x_{n}-x_{\text {neck }}\right)^{2}+\mathcal{O}\left(x_{n}^{3}\right),
$$

with $\kappa=\left.\frac{1}{2}\left(\partial^{2} f\left(x_{n}\right) / \partial x_{n}^{2}\right)\right|_{x_{\text {neck }}}$ and neck width $\varepsilon=f\left(x_{\text {neck }}\right)-x_{\text {neck }}=\dot{x}\left(x_{\text {neck }}\right) \mathrm{d} t$. Calculating the extrema of (2.12) we find for $x_{\text {neck }}$ 


$$
x_{\text {neck }}=\frac{1}{2} \pm \frac{1}{2 \pi} \arccos \left(\frac{B}{\pi}\right)-\varphi+m \quad\left(\begin{array}{c}
+\max \\
-\min
\end{array}\right), \quad m \in \mathbb{Z},
$$

which gives

$$
\dot{x}\left(x_{\text {neck }}\right)=C_{ \pm}(B)+2 B(\varphi+m)+E,
$$

with $C_{+}(B)$ a constant depending on $B$ and on whether the extremum in (2.17) is a $\max$ or min. So we see that the neckwidth $\varepsilon$ depends linearly on the pinning phase $\varphi$, as well as the field $E$. Availing ourselves of the scaling result of Pomeau and Manneville [16], this leads to the following relation for $\tau$ in terms of $\varphi$ :

$$
\tau(\varphi) \sim\left|C_{1}(E, B)+C_{2}(E, B) \varphi\right|^{-1 / 2},
$$

where the $C_{i}$ depend only on $B$ and $E$.

We may now write down the $\tau$-distribution in terms of the distribution of pinning phases,

$$
\mathcal{N}_{\tau}(\tau) \sim \int \mathrm{d} \varphi \mathcal{N}_{\varphi}(\varphi) \delta\left(\tau-\left(C_{1}+C_{2} \varphi\right)^{-1 / 2}\right),
$$

which gives, for a uniform distribution $\mathcal{N}_{\varphi}$ over a finite interval,

$$
\mathcal{N}_{\tau}(\tau) \sim \tau^{-3}
$$

after a standard substitution of variables. Note that in the absence of randomness in the $\varphi$ (or $E$ ), there will be but one characteristic waiting time. Then, after exhibiting a single peak, the current decays exponentially, with the decay rate given by the Lyapunov exponent of the map (2.10).

\section{Simulation results}

\subsection{Summary}

In this section we want to present the numerical work done on the system of coupled maps defined in section 2 . We will first outline some technical details and make general remarks about the simulations. We found three different classes of relaxation behaviour.

For an ensemble of single-particle maps (2.10), obtained from the $\mathrm{N}$ dimensional equations of motion (2.6) by treating the nearest neighbors of 
each dynamical variable as if they remained fixed, we reproduced the passage time distributions we predicted on the basis of scaling properties of intermittent maps, with and without external noise [16-18]. The distribution of different characteristic relaxation times thus obtained lead to a power-law decay, universal with respect to field and coupling constant, up to corrections to scaling.

For the system of coupled maps (2.6) with uniform pinning ( $\varphi=$ const), we find classical exponential relaxation below threshold. This is drastically changed when configurational noise is added and yields stretched exponential relaxation.

For the coupled map (2.6) with random pinning, the relaxation obeys a stretched exponential law, $\sim \exp \left(-t^{\beta}\right)$. We performed extensive simulations to obtain the exponent $\beta$ as a function of the field $E$. We found a general lowerbound $\beta_{\min } \approx 0.3$, and a peak $\beta_{\max } \approx 0.5$ for $0.55<E<0.60$. The coupled map exhibits a dynamical phase transition [20] parametrized by the field $E$ (for fixed coupling constant $B$ ) from relaxation mode (pinned) to steady state (depinned). For $B=1$, the sample dependent threshold field is approximately at $E_{\mathrm{th}}=0.70$. In the neighborhood of the threshold, the system displays self-similarity and $1 / f$ noise.

Although in the pinned region the spatial correlations in this system are found to be very short (of the order of the lattice spacing), the effect of the elastic coupling is highly non-trivial. Attempts at mimicking the local field due to the nearest neighbors by several time-dependent noise terms, with or without memory, all yielded behaviour qualitatively similar to that of ensembles of single-particle maps, namely, power law decay. Comparing these results with similar behaviour obtained by more sophisticated single particle approaches $[14,15]$ seems to suggest that the system is inherently of high effective dimensionality [10] and cannot be treated satisfactorily by few-effectivedegrees-of-freedom approaches.

\subsection{Numerical methods}

All programs were written in Pascal and executed on a VAX 8650 and Cyber $170 / 760$ at the Rijksuniversiteit Groningen. A typical job consisted of a series of 10 to 50 separate simulations with different random initial conditions. For each of them the normalized current $J(n) / J(0)$ and the waiting time distribution $\mathcal{N}_{\tau}(n)$ were computed, after which the averaged results were stored. To compute the waiting time distribution, at every timestep $n$ each site $i$ was checked for a change of sign in the second time-derivative of $\psi$. If a change of sign occurred and the velocity $|\dot{\psi}|$ exceeded a threshold $a(a=0.001)$, the integer $\mathcal{N}_{ \pm}(n)$ was raised by 1 ( \pm depending on the sign of $\dot{\psi}$ ).

The initial configurations for $\varphi$ and $\psi$ were taken from a uniform distribution 
between 0 and 1 since this covers a complete period of the sine. Better statistics would be obtained by performing a pre-relaxation with zero field before the actual simulation takes place. Then, turning on the field would only induce positive transitions and $\mathcal{N}_{\tau}(n)=\mathcal{N}_{+} \sim J$. But since this method causes a doubling of CPU-time we omitted the pre-relaxation. The price we pay for this is that, as can be seen from fig. 3 , we will be measuring also negative events, contributing to $\mathcal{N}_{-}$. Particularly for small fields $E$ this causes a slight distortion in the measured current $J$, which is then $J(n) \approx \mathcal{N}_{+}(n)-\mathcal{N}_{-}(n)$.

We checked that our results did not depend on our choice of $\mathrm{d} t$. Graphs and fits for $\mathrm{d} t=0.01$ and $\mathrm{d} t=0.1$ reveal no significant differences either upon inspection or by comparison of fitting parameters and the latter only uses $\frac{1}{10}$ of the time needed by $\mathrm{d} t=0.01$. So most simulations were performed with $\mathrm{d} t=0.1$, unless specified otherwise.

The regimes of interest for the field $E$ and coupling constant $B$ could be determined with help of the rough phase diagram provided by Pietronero and Strässler [6,7]. Although we limited ourselves to probing the field dependence of various quantities of interest, at fixed $B=1$, it would obviously be desirable to also investigate the effect of varying $B$ at fixed $E$.

Long chains of $10^{4}$ sites were used except when stated otherwise. A large sample exhibits a longer lasting relaxation because one statistically has a larger probability to encounter very large waiting times. We used large samples because the averaged dataset is as large as the simulation which is cut off the earliest, and we implemented a flexible stopping condition which stopped the simulation when entering the numerical noise zone for the current $J$. To get rid of end-effects cyclic boundary conditions were used.

We fitted the datasets with power laws, exponentials and stretched exponentials using fitting routines based on the method of least squares. The datasets were, where possible, manipulated in such a way that the actual fitting could be done with a straight line. Moreover, we restored the equal spacing of the independent variables by either choosing an equidistant set of data points, or choosing the midpoints of equal intervals over which the dependent variables were averaged. For a stretched exponential of the form

$$
y(x)=a \exp \left(c x^{b}\right)=y(0) \exp \left(c x^{b}\right)
$$

one can compute the double logarithm

$$
\ln [\ln y(0)-\ln y(n)]=\ln c+b \ln x .
$$

But as one can see in (3.1) the amplitude $y(0)$ appears as a parameter of the fit, and one is only allowed to take it equal to the numerically obtained initial 
value if one indeed expects stretched exponential behaviour starting from very small times, and there are no distortions due to transient effects. Since all of our fits hold only for intermediate times we have to do a three-parameter fit of the following kind:

$$
\hat{y}_{i}=A-C x_{i}^{B}
$$

Formula (3.3) proved to be very sensitive to the inevitable scatter of the experimental datasets but at least not as much as a fit of the stretched exponential of (3.1) (with free parameters $a, b$, and $c$ ). This problem is eliminated in case it is possible to choose one of the parameters in (3.1) or (3.3) to be fixed. for the details we refer to the sections in which we treat the actual fits.

\subsection{Single-particle relaxation}

For an ensemble of effectively uncoupled single-particle maps (2.10) we derived the waiting time distribution analytically at the end of section 2 . In this section we will compare these results with the simulations. We predicted that with uniform pinning we should find a delta function for the waiting time distribution, and for random pinning a power law,

$$
\mathcal{N}_{\tau}(\tau) \sim \tau^{-\sigma}, \quad \sigma=3,
$$

as well as $J(t) \sim t^{-\sigma}$. In the presence of a vanishingly small field and $B=1$, the powers with which $\mathcal{N}_{\tau}$ and $J$ decay are found to be $\sigma_{\tau}=2.90^{ \pm 0.10}$ and $\sigma_{J}=$ $2.94^{ \pm 0.10}$ respectively (see fig. 6). For higher fields $E$, one finds $2.70<\sigma<2.90$. This is probably due to corrections to scaling, which one should expect for a finite neck $[17,18]$.

Hirsch et al. have performed simulations on the mean passage time for the three-fold iterate of the logistic map (which yields a form like (2.16) if one expands around the point of near tangency). They also did simulations on (2.16) in the presence of additive time-dependent Gaussian white noise $\xi(t)$,

$$
\langle\xi\rangle=0, \quad\left\langle\xi^{2}\right\rangle=1, \quad\left\langle\xi(t) \xi\left(t^{\prime}\right)\right\rangle=\delta\left(t-t^{\prime}\right),
$$

and obtained a power-like tail for the path length distribution. Since the random pinning is responsible for a power law tail in (3.4) we made a simple approximation to see if we could mimic effect of random pinning with a linear additive configurational noise term. Expanding the pinning term in (2.10) 


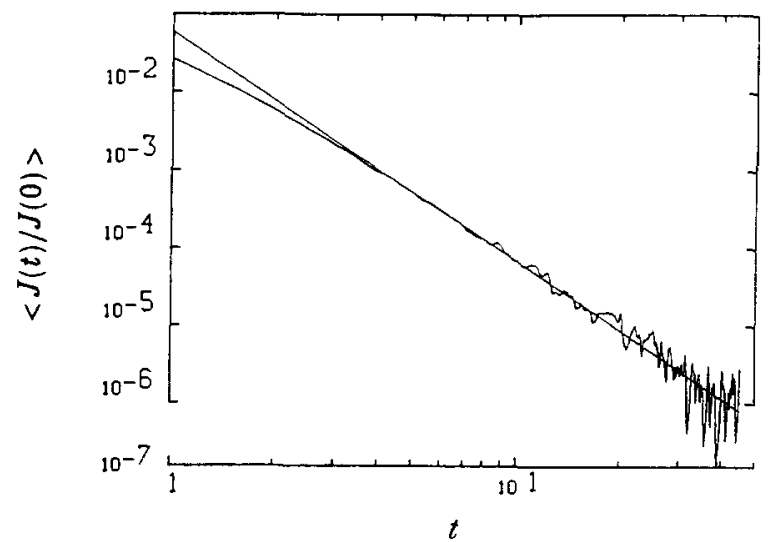

Fig. 6. Fit of the current for an ensemble of uncoupled maps $(E=0, B=1$, random pinning, 50 samples) with power law exponent $\beta=2.94^{ \pm 0.10}$.

around $\psi_{j}$, we get

$$
\sin \left\{2 \pi\left[\psi_{j}(n)+\varphi_{j}\right]\right\} \approx \sin \left[2 \pi \psi_{j}(n)\right]+\varphi_{j} \quad \text { with }-1<\varphi_{j}<1
$$

where the $\varphi_{j}$ are constant in time. In fig. 7 we plotted the distribution of waiting times we obtain with the approximation (3.6). The resemblence to fig. 15 of Hirsch et al. [17] is striking. For $\varphi=0$ (uniform pinning) we find a sharp peak, as we predicted, and with the noise term on we get the power-like tail. We have fitted this tail and we found a power $\sigma \approx 2.70$ (see fig. 8 ). We find it remarkable that the effect of configurational randomness on the waiting time distribution is the same as that of time-dependent additive noise.

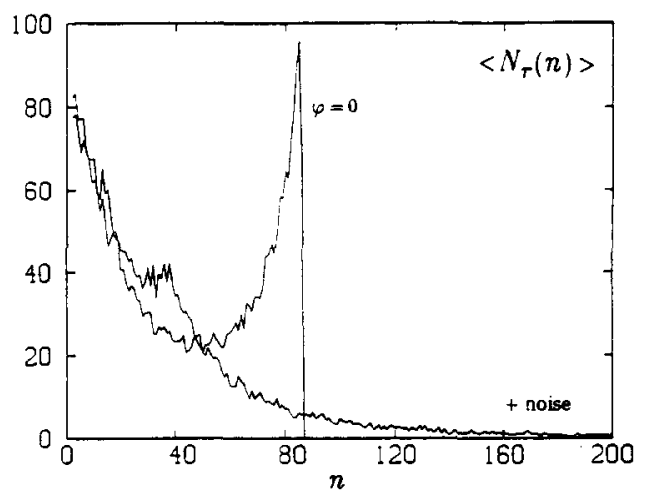

Fig. 7. Waiting time distribution for an ensemble of uncoupled maps with uniform pinning ( $E=0$, $B=1, \varphi=0,10$ samples). An added noise term yields a power law tail. 


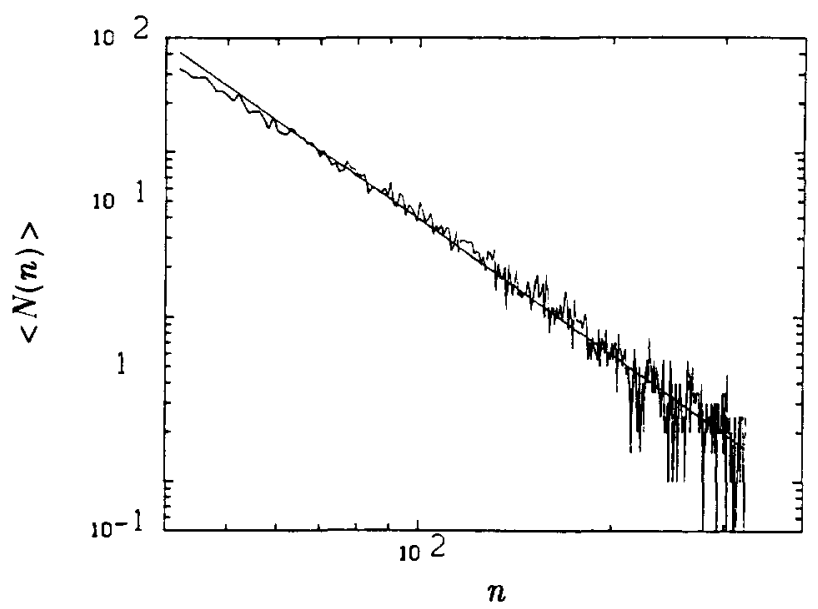

Fig. 8. Power law fit of the tail of the waiting time distribution as presented in fig. 7 yielding an exponent $\beta=2.72 \pm 0.10$.

Finally, we tried to see if we could simulate the effect of the coupling term in eq. (2.6) by treating the $E_{\text {eff }}$ in (2.9) as a time dependent noise term, with or without memory. In the coupling term in (2.6) we substituted

$$
\begin{aligned}
{\left[\psi_{j-1}(n)+\psi_{j+1}(n)\right] \approx } & p\left[\psi_{j-1}(0)+\psi_{j+1}(0)\right]+2(1-p) P(n-1) \\
& +\alpha \frac{1}{n} \sum_{j}^{n} \xi(j)
\end{aligned}
$$

where $p$ and $\alpha$ are adjustable parameters, with $P(n-1)=\left\langle\psi_{j}(n-1)\right\rangle$ the total polarization at time $n-1$ and $\xi$ a time dependent noise term (as in (3.5)) with its fluctuations smoothed out by the average over $n$. But the stretched exponential relaxation behaviour observed for coupled maps in the presence of configurational randomness could not be reproduced in this manner for any combination of $p$ or $\alpha$. We obtained only power laws with $\sigma$ in the range $2.5<\sigma<3.5$. Thus the time dependence of the effective field due to the neighbors is highly non-trivial and cannot be mimicked by a noise term. This implies that the effective high-dimensional dynamics of the full model (2.6) cannot be reduced to a few degrees of freedom model such as an ensemble of maps given by (2.10).

\section{Coupled maps with uniform pinning}

In this section we present numerical results from simulations of the coupled map lattice (2.6) with uniform pinning, namely, $\varphi_{j}=$ const for all sites $j$. For 
most runs the constant was taken equal to zero. No essential differences were found with the runs we did for comparison with const $\neq 0$.

With uniform pinning in the relatively strong pinning region $(B=1)$ we have investigated, one has a unique equilibrium state and all the $\psi_{j}$ go to the same fixed point. We found exponential relaxation of the type

$$
J(t) \sim \exp \left(-t / \tau_{0}\right)
$$

for $E<E_{\mathrm{th}} \sim 1$, with $\tau_{0}$ decreasing with increasing $B$ or $E$. The waiting time distribution has the same form as the current (see figs. 8 and 9). The initial differences between the phases $\psi_{j}$ (from the starting configuration) stay constant till they one by one reach the same fixed point. If, on the other hand, the system is allowed to reach equilibrium at zero field, this causes all the $\psi$ 's to "walk in line" when the field is turned on.

For low fields $(E \leqslant 0.3)$ the current $J$ decays too fast and too irregularly to allow a determination of its functional form. For higher fields, the current displays noisy oscillations superimposed on the exponential decay which evens out when $E$ is increased $(0.5 \rightarrow E \rightarrow 0.9)$, till the threshold field $E_{\mathrm{th}} \approx 1$ is reached. An explanation for this oscillation is to be found in the field of the washboard potential. Above $E_{\mathrm{th}}$ we find an oscillating current with a single characteristic frequency and a nonzero average. Right at threshold the current obeys a power law $\sim t^{-2}$ (see fig. 10).

Finally, as in the previous section, we were able to mimic the effect of random pinning by perturbing the coupled map lattice with a linear additive noise term. In fig. 11, we see the current plot for a simulation of a coupled map with uniform pinning but with added noise term $\mathrm{d} t \varphi_{j}$ and $-0.1<\varphi_{j}<0.1$. This should be compared with fig. 2 .
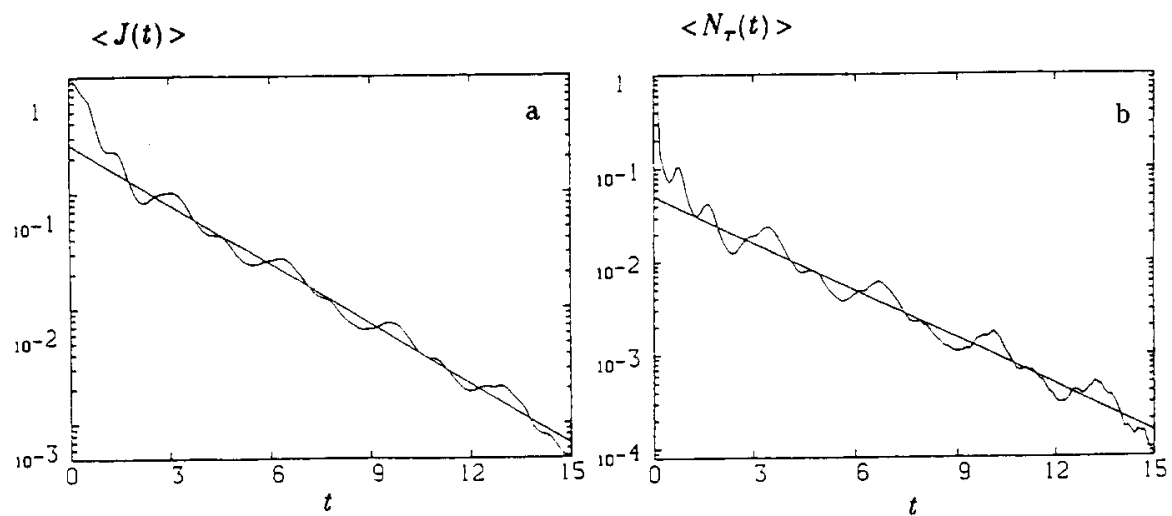

Fig. 9. Exponential fit of (a) the current and (b) the waiting time distribution for a system of coupled maps with uniform pinning $\left(E=0.5, B=1, \varphi=0\right.$, 50 samples) both yielding $\tau_{0} \sim 2.50$. 


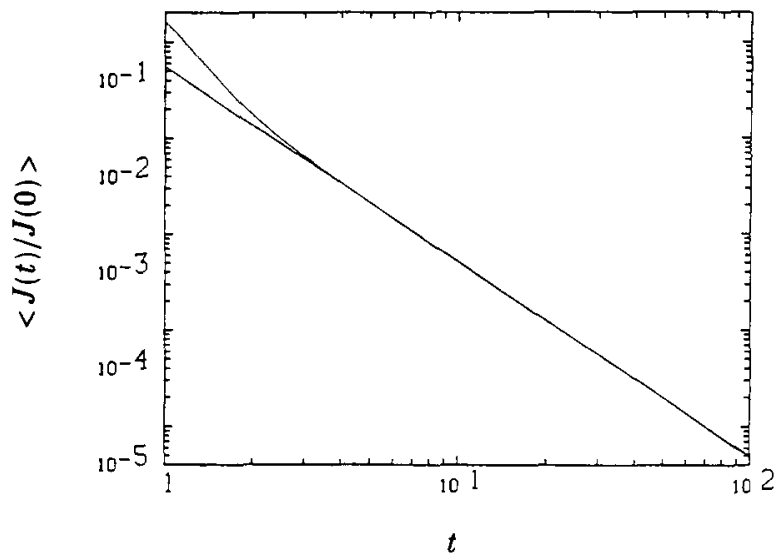

Fig. 10. Power law fit of the current at threshold field for a system of coupled maps with uniform pinning ( $E=1, B=1, \varphi=0,50$ samples) yielding $\sigma=2.00$.

\subsection{Coupled maps with random pinning}

In this section we report our numerical results for the complete coupled map lattice (2.6).

The average current $J$ for a number of different fields $E$ below threshold is shown in fig. 12. These curves are very well represented by a stretched exponential form,

$$
J(t) \sim \exp \left(-t^{-\beta}\right) .
$$

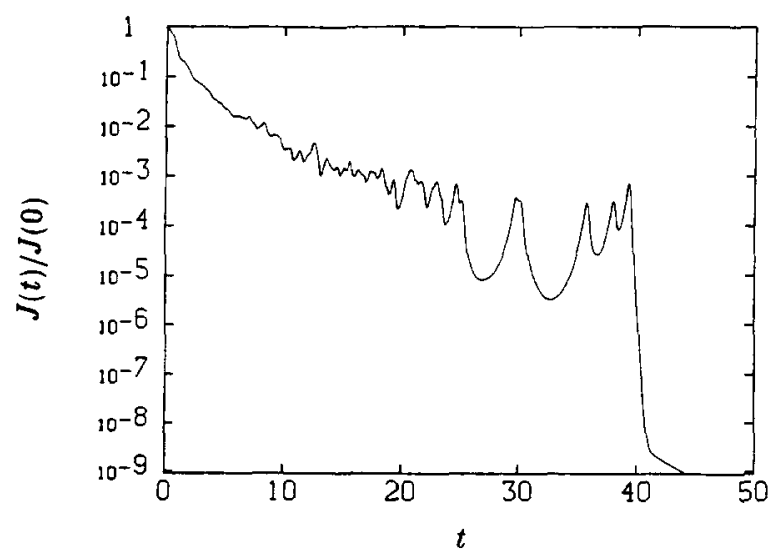

Fig. 11. Current for the coupled map with uniform pinning $(E=0.5, B=1, \varphi=0)$ but with added noise term to mimic random pinning. 


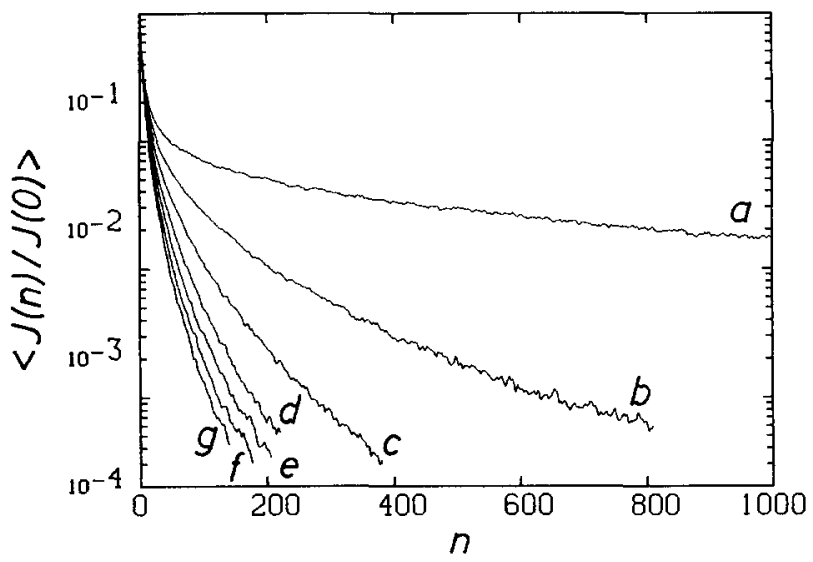

Fig. 12. Current for coupled maps with random pinning and various fields ( $B=1,50$ samples). The various curves refer to: (a) $E=0.70 \simeq E_{\mathrm{th}}$; (b) $E=0.65$; (c) $E=0.60$; (d) $E=0.55$; (e) $E=0.50$; (f) $E=0.45$; (g) $E=0.40$.

We plotted our estimates for the stretched exponential exponent $\beta$ for fixed $B$ as a function of the field $E$ in fig. 13. Despite the rather large error bars, whose origin we will discuss below, its mean features are quite clear. We find a minimum $\beta_{\min }=1 / 3$, an increasing $\beta$ with increasing $E$, a maximum between $0.5<E<0.6$, and from about $E=0.6$ till $E=E_{\mathrm{th}} \approx 0.7$ a decreasing $\beta$.

We have pointed out in section 3.2 that the three-parameter fitting formula (3.1) appeared to very sensitive to changing the lower and, to a lesser extent, the upperbounds $\left(t_{1}, t_{2}\right)$ of the time interval over which the fit was performed. Since the relaxation behaviour will not be stretched exponential, but rather exponential, for very small and very large $t$, we had to use fitting programs that

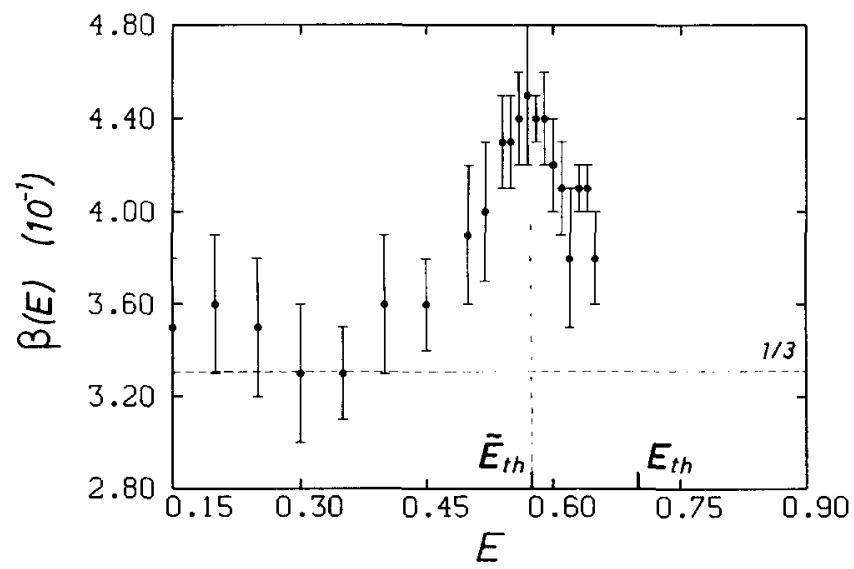

Fig. 13. Stretched exponential exponent $\beta$ plotted as a function of the field $E$. Each point stands for $50-100$ runs. 
scanned a dataset for $t_{1}$ (and sometimes $t_{2}$ ). The error bars for $\beta$ arise from the range over which the exponent fluctuated as a function of $t_{1}$.

For large $t$ one only finds single events with rather large gaps in between (see fig. 2), so one could stop iterating if $J$ falls below a certain $J_{\min }$. This will automatically cut off this single-event zone but sometimes too much. If, for instance, 1 out of the 100 samples just happens to exhibit such a dip at an earlier stage than the bulk, it will determine $t_{2}$ for the average. The long tailed distributions we deal with ensure that the more samples one averages over the more likely it is that such "bad luck" will occur.

For very short times the current is dominated by the movement of the sites which relax exponentially towards nearby fixed points without having to iterate through a neck region on their trajectory. For some low field simulations we indeed found, for the very first iterations, exponential decay. On the other hand, one can see clearly that a high field means a lot of events, i.e. good statistics, which implies a rather smooth curve over a longer range. Therefore we can report higher accuracy for high field relaxation.

It should be stressed that the fits which lead to fig. 13 individually look very good (see fig. 14 for an example), and yield error bars for the free parameters, which are of the order of 1 percent. A stretched exponential fit with three parameters according to (3.1) can find combinations $(a, b, c)$ which minimize the total errors with respect to the dataset almost as well as other combinations. As soon as one is fixed, the other two stay almost constant with respect to a changing fitting regime. However, there is no obvious way in which one of the parameters could be thus eliminated. With fig. 15 we provide an example of what might happen if one cautiously would fit with (3.2).

At $B=1$ the threshold field $E_{\mathrm{th}}$ is about 0.7 , depending on the pinning

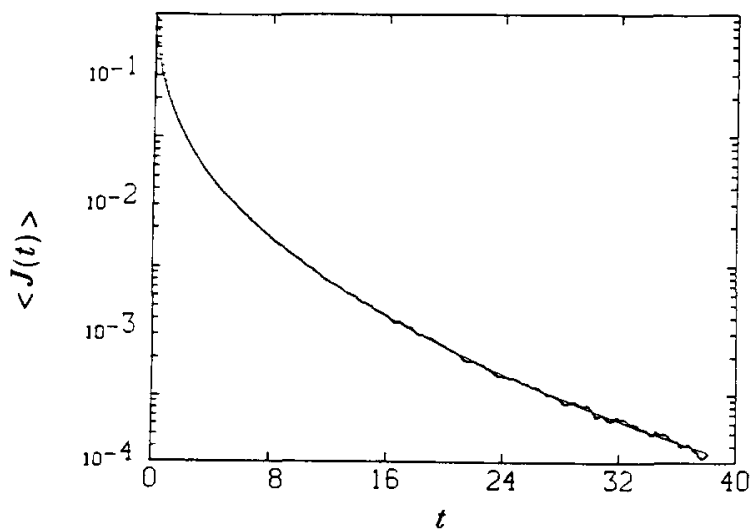

Fig. 14. An example of a stretched exponential fit of the current for a coupled map with random pinning ( $E=0.6, B=1,100$ samples) giving $\beta=0.42$. 


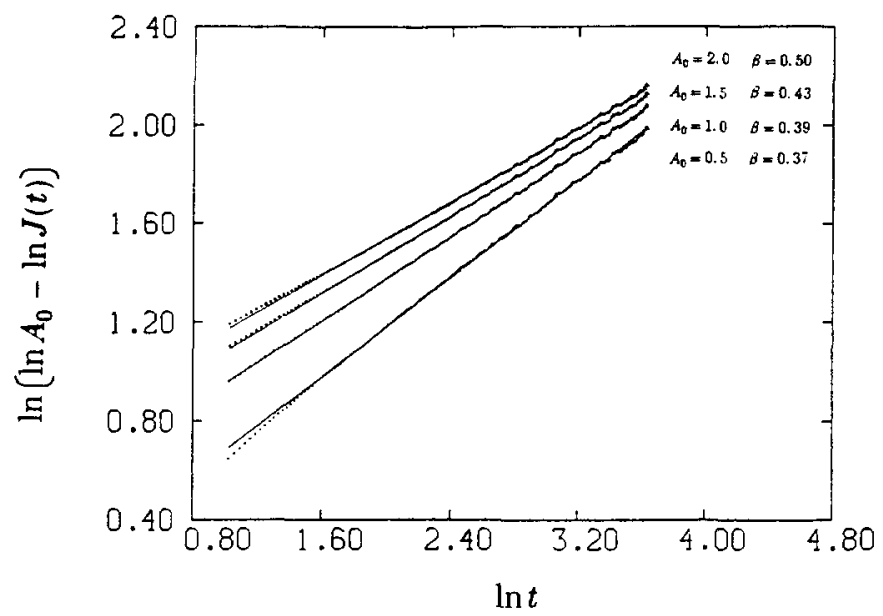

Fig. 15. Double-log fits to the same dataset as used in fig. 14 with different initial amplitudes $A$ $(=y(0)$ in eq. (3.2)). By adjusting the lower bound each fit would look satisfactory.

configuration. Right at threshold, a fit stretching out over the first decades indicates a power law dependence for the current but we cannot confirm this for the complete range of data that we obtained from the simulations. The deviations from a power law for large times may be due to finite size effects, but this remains to be investigated.

For large $E$ and large $B$, that is, in the unpinned region, we find an oscillating current which is non-decaying. The power spectrum $S(k)$ displays both narrow and broad band noise (see fig. 16), and contrary to $E \leqslant E_{\mathrm{th}}$ we

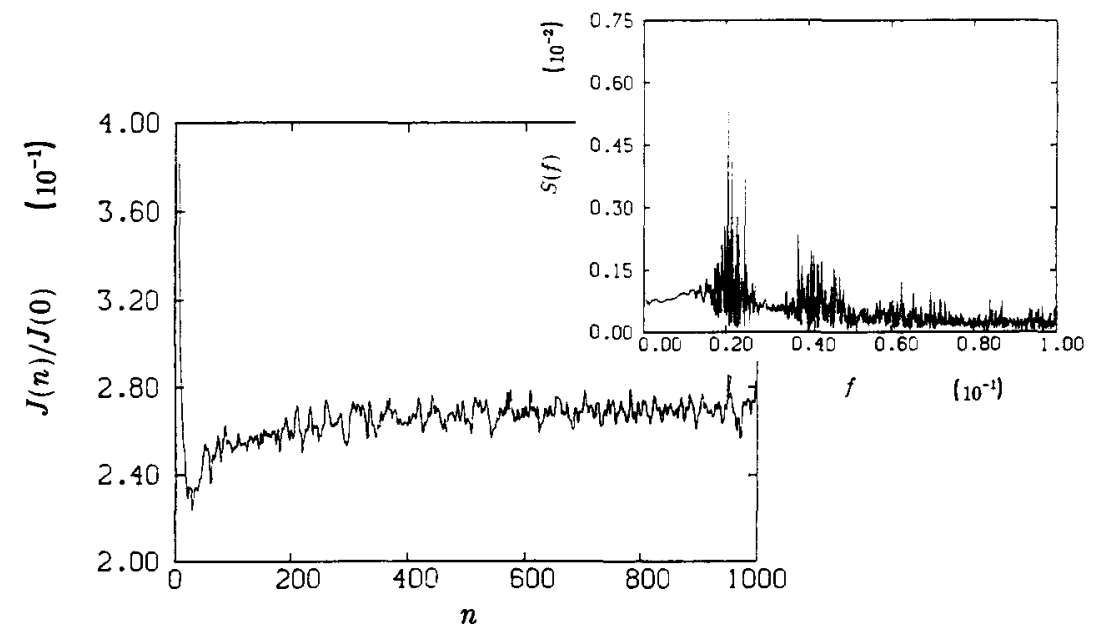

Fig. 16. Non-decaying current for a coupled map with random pinning in high field ( $E=0.8>E_{\mathrm{th}}$, $B=1$ ) with the power spectrum displaying both narrow and broad band noise. 


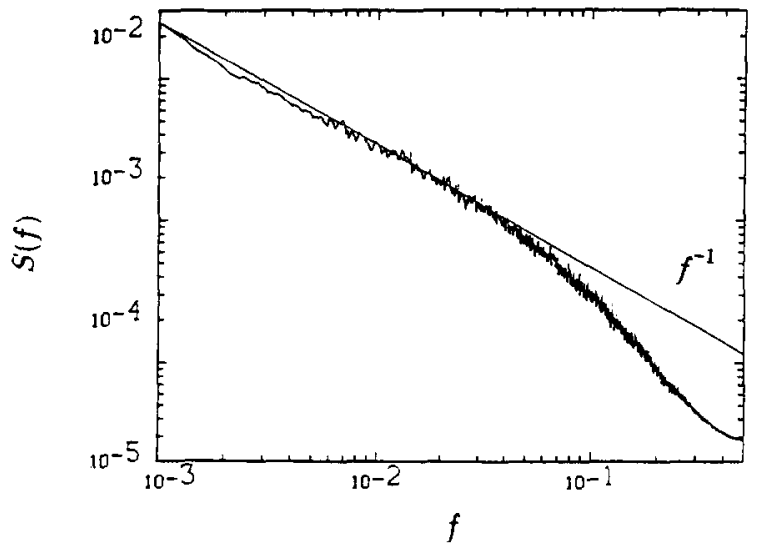

Fig. 17. Power spectrum of the current reflecting $1 / f$ noise at threshold field for a coupled map system with random pinning $\left(E=0.7 \sim E_{\mathrm{th}}, B=1\right)$.

find a large peak for $S(k=0)$. This is in agreement with previously reported results [6]. For the power spectrum in the pinned regime $\left(E<E_{\mathrm{th}}\right)$ we found a power law $\left(f^{-\phi}\right)$ with exponent $\phi$ close to $2(1.8<\phi<2.0)$, this implying Guassian fluctuations rather than $1 / f$ noise. However, right at the threshold field $\left(E=E_{\mathrm{th}}\right)$, we did find $1 / f$ behaviour $(\phi \approx 1)$ in the low frequencies (see fig. 17).

A cellular automaton visualization of the velocity profiles (see section 4) shows that a relatively short chain (down to $N=5$ !) with a given configuration of pinning phases undergoes the same microscopic relaxation process as when it is embedded in a longer chain, even at the endpoints [21]. This suggests that the spatial correlations are very weak even when the system is performing completely coherent periodic motion in time, as already remarked by Littlewood [8]. Indeed, a numerical computation of the correlation function

$$
C_{t}(r)=\left\langle\psi_{i} \psi_{i+r}\right\rangle /\left\langle\psi_{i}\right\rangle\left\langle\psi_{i+r}\right\rangle
$$

revealed spatial correlations in $\psi_{j}$ in the pinning regime to be of the order of a lattice spacing. The Fourier transform of $C_{t}(r)$ has a Gaussian like envelope, centered around $k=0$.

\section{Spatio-temporal patterns}

Coupled map lattices, similar to that in (2.6), are a growing area of research $[11,12]$, promising to a new testing ground for models and ideas on chaos. Variants of the CMP in (2.6) have been investigated by Kaneko [11], and 
shown to give rise to spatio-temporal patterns exhibiting intermittent and chaotic behaviour.

In this section we will make use of a simple cellular automaton representation [11] in order to display the spatio-temporal distribution of the individual relaxation events (see section 2) or abrupt changes exhibited by the phase variables, while the system represented by (2.6) evolves in time. The patterns thus formed are found to be self-similar, with a well-defined fractal dimension, right at the threshold, while above and below no such self-similarity is observed.

The cellular automaton representation is defined as follows:

$$
S_{j}(n)= \begin{cases}0, & \dot{\psi}_{j}(n)<a, \\ 1, & \dot{\psi}_{j}(n) \geqslant a,\end{cases}
$$

where $\dot{\psi}_{j}(n)=\psi_{j}(n+1)-\psi_{j}(n)$. For each time step $n$ and $j=1, \ldots, N, S_{j}(n)$ is computed from (2.6) and (4.1). Plotting dots where $S_{j}=1$ and blanks otherwise, yields patterns such as those in fig. 18. Clearly, $S_{j}(n)=1$ at those $n$ where $\psi_{j}$ makes an abrupt transition.

An inspection of the plots in fig. 18 suggests that for relatively low fields (e.g.
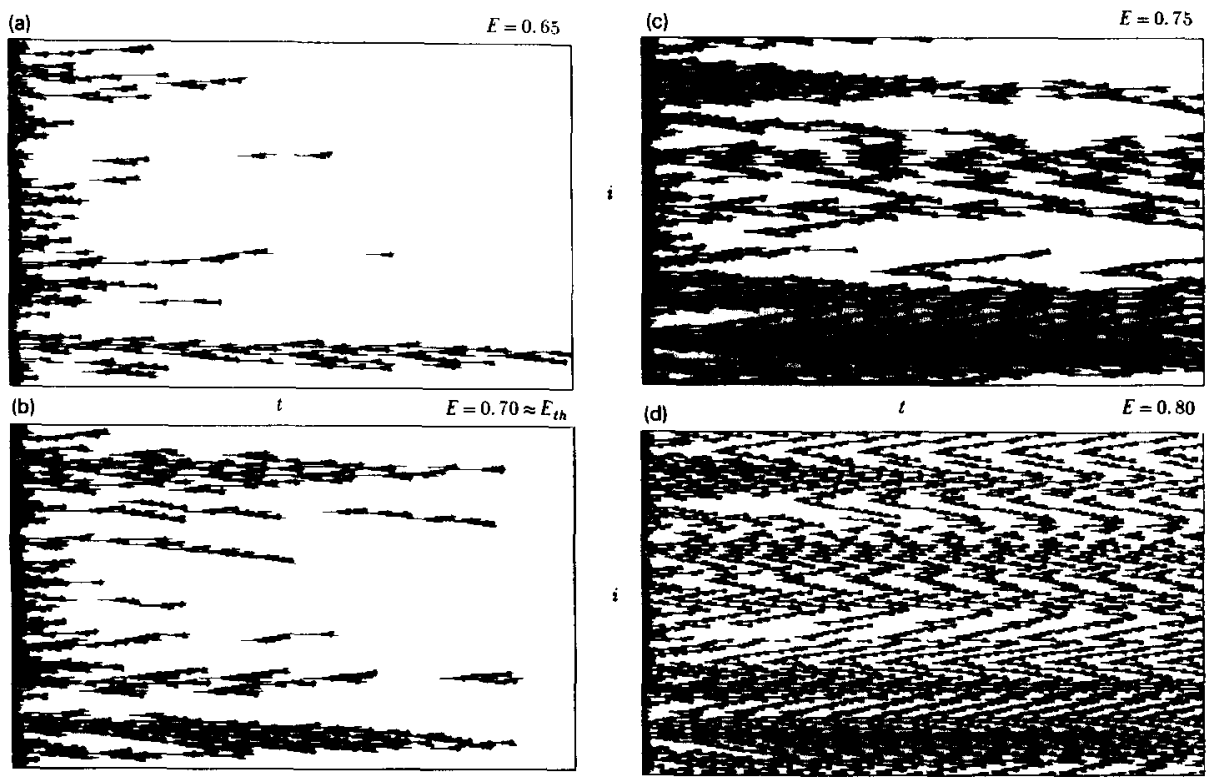

Fig. 18. Realizations of the cellular automaton representation of the randomly pinned CDW (see rule (4.1)). The system size if 512 and 1024 time steps have been taken. The lower cutoff in the velocity, $a$, is $a=0.002$ except for $E=0.80$, where it is 0.01 . The vertical axis corresponds to the positions along the chain and the horizontal axis represents time. 

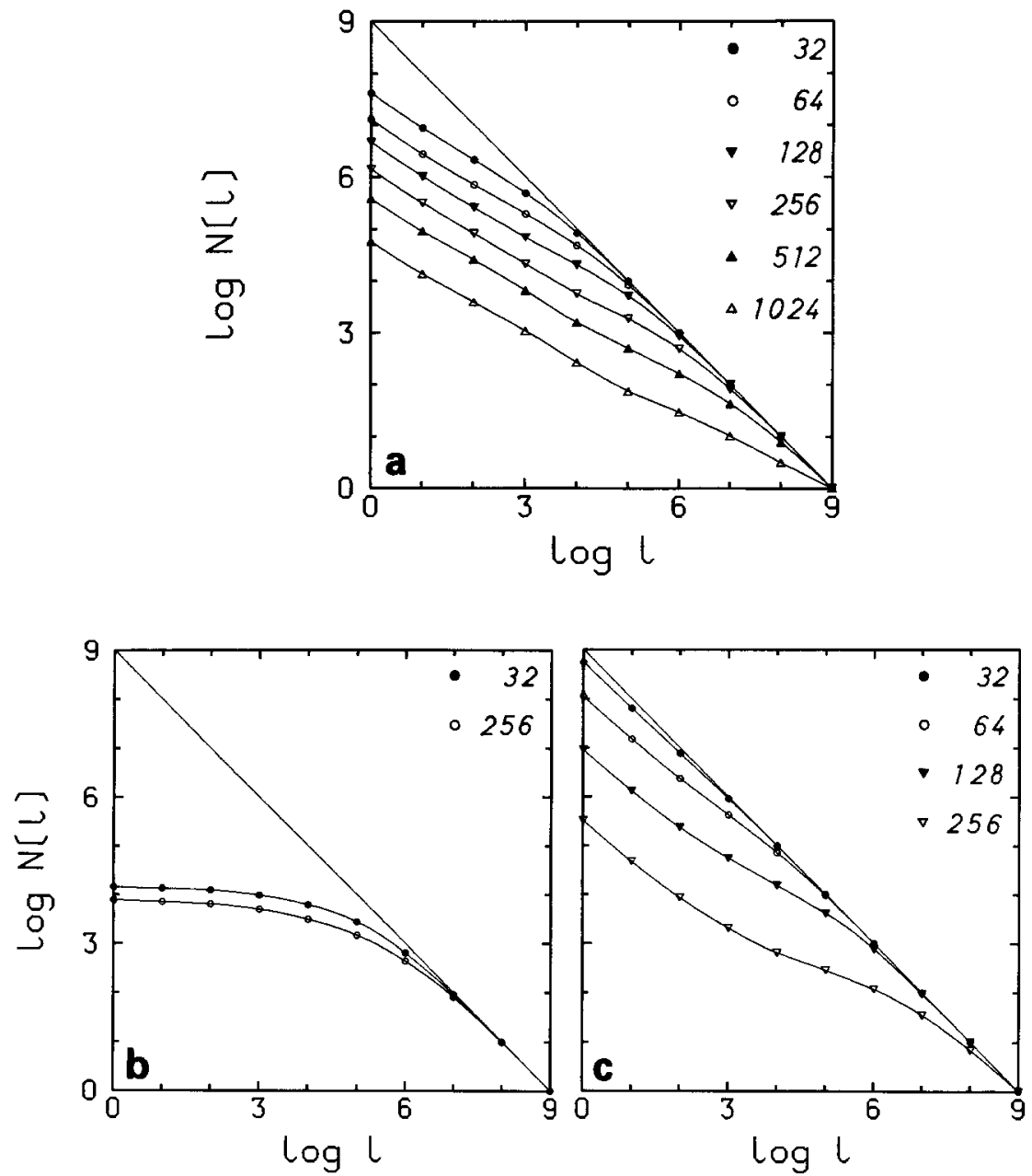

Fig. 19. The logarithm $m_{2}$ of the mass versus the length scale at constant time for the spatiotemporal patterns in fig. 18. The mass points have been averaged over 100 realizations. $E=0.70$, 0.75 and 0.65 in (a), (b) and (c), respectively.

$E=0.65)$ the spatio-temporal correlations between the events are small, whereas above threshold one clearly discerns quasi-periodic motion fully correlated in time.

We have performed box counting to determine the scaling behaviour of the total "mass" (sites in motion showing up as occupied dots) at each time step $n$. For each separate field, averages were performed over 100 samples. At a given $n$, the number of occupied "boxes" $N(n, m)$ at each length scale $l=2^{m}$ was averaged over the 100 realizations. Our results are shown in fig. 19 for different $E$ at fixed $B=1$. The points fail to fall on straight lines for $E<E_{\mathrm{th}}$ and 
$E>E_{\mathrm{th}}$, the limiting slope for small box sizes being 1 and 0 , respectively. This can be understood from a close inspection of fig. 18. In the former case the mass formes compact fingers and in the latter it is organized in straight line segments. For large scales the slope tends in these cases to 1 . At the threshold, on the other hand, although the mass decreases with time, the scaling behaviour is remarkably uniform, and extends over five to six decades. The fractal dimension $D_{\mathrm{f}}+0.58 \pm 0.01$. We checked that varying the lower cutoff $a$ did not affect the results, except to shift the scaling regime.

The scale invariance and fractality of the "active" region at threshold is a manifestation of the dynamical phase transition [20] that takes place there. Similar patterns consisting of all the points that make transitions until an initial perturbation (here turning on the field) completely subsides, have been investigated by Bak et al. [22]. They found such self-similar patterns in the neighborhood of a "critical state", which was a dynamical attractor, whereas in the present case this state is achieved by adjusting the parameter $E$.

For the sake of completeness, we have also investigated the CML in (2.6) with an added non-linearity. After Kaneko [11] we write

$$
x_{j}(n+1)=h\left(x_{j}(n)\right)+\frac{1}{2} \varepsilon\left[g\left(x_{j+1}(n)\right)+g\left(x_{j-1}(n)\right)-2 g\left(x_{j}(n)\right)\right],
$$

with $j=1,2, \ldots, N$ and periodic boundary conditions and

$$
h(x)=|x+A \sin (2 \pi x)+C|(\bmod 1), \quad \text { with } A=0.2, \quad C=0.55,
$$

The circle map $h(x)$ exhibits a stable cycle with period two: $x_{n+1}^{*}=h^{2}\left(x_{n}^{*}\right)$. The parameters are chosen such that a slight perturbation will yield the intermittent transition of Pomeau-Manneville [16]. By choosing a small coupling constant $\varepsilon$ in (4.2), such a small perturbation is provided by the coupling part. The patterns of bursts and laminar clusters (see fig. 20) are obtained by a similar

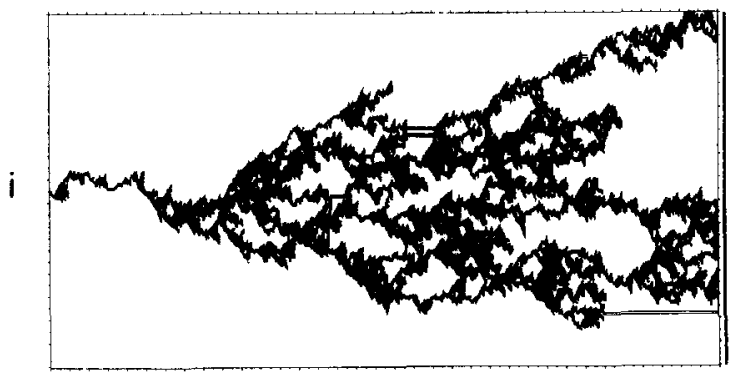

$32 n$

Fig. 20. Realization of a kink pattern from the cellular automaton representation (4.3) of the coupled map lattice (4.2) with initial conditions (4.4). See text. 
plotting criterion as we used for the velocity profiles, where we measured temporal differences, while here "kinks" or abrupt spatial differences are registered according to the following rule:

$$
S_{j}(n)=\left\{\begin{array} { l } 
{ 1 } \\
{ 0 }
\end{array} \quad \text { for } | x _ { j + 1 } ( n ) - x _ { j } ( n ) | ( \operatorname { m o d } 1 ) \left\{\begin{array}{l}
>\delta \\
\leqslant \delta
\end{array} .\right.\right.
$$

Random initial conditions $\left(0 \leqslant x_{j}<1\right)$ can be used, or single kinks implemented by putting half of the chain equal to the fixed point $x_{1}^{*}$ and the other half to $x_{2}^{*}$,

$$
\begin{array}{ll}
x_{j}=x_{1}^{*} & \text { for } 1 \leqslant j \leqslant N / 2, \\
x_{j}=x_{2}^{*} & \text { for } N / 2<j \leqslant N .
\end{array}
$$

Since $x_{2}^{*}=h\left(x_{1}^{*}\right)$ and $x_{1}^{*}=h\left(x_{2}^{*}\right)$ the sites keep alternating between $x_{1}^{*}$ and $x_{2}^{*}$ and $x_{j+1}-x_{j}$ will be zero except at the kinks which are initially positioned at $N$ and $N / 2$. Right at these kinks the motion is chaotic and there are large differences between neighbouring sites. In this way one can follow the propagation of the kinks in time and space. In fig. 20 we give an example for a representation (4.3) of the coupled map (4.2) with initial conditions (4.4). We used absorbing boundary conditions and applied the $(\bmod 1)$ operator on the complete map (4.2) instead of only on the circle map, which is a minor change to the original implementation. The white regions correspond to laminar zones where sites alternate regularly between the two fixed points; the black parts correspond to chaotic bursts. Lowering the coupling confines the region of propagation and reveals patterns looking like the growth of dendritic crystals, increasing the coupling reveals a kind of "fully developed turbulence". The parameters for fig. 20 are $\mathrm{d} t=A=0.2, E=C / \mathrm{d} t=2.75$ and $B=\varepsilon / 2 \mathrm{~d} t=$ 0.595 .

Although for any given time $n$ this pattern shows excellent mass to length scaling, the fractal dimension depends on $n$, decaying slowly with time.

It is interesting to note that defining a Boolean variable $\tilde{x}=\left(0\right.$ for state $x_{1}^{*}, 1$ for state $\left.x_{2}^{*}\right)$ and mimicking $h(x)$ by $F(\tilde{x})=(\tilde{x}+1)(\bmod 2)$, yields the cellular automaton

$$
\tilde{x}_{j}(n+1)=\left[F\left(\tilde{x}_{j}(n)\right)+\tilde{x}_{j+1}(n)+\tilde{x}_{j-1}(n)-2 \tilde{x}_{j}(n)\right](\bmod 2),
$$

which is "illegal" according to the rules of Wolfram [23]. However, it is easy to see that for the "kink" variables defined via

$$
\tilde{S}_{j}(n)=\theta\left(\left|\tilde{x}_{j+1}(n)-\tilde{x}_{j}(n)\right|\right)
$$


one has a legal cellular automaton giving rise to a fractal pattern and identified as "rule 150 " in Wolfram's scheme [23].

\section{Acknowledgements}

A. Erzan would like to thank the "Stichting voor Fundementeel Onderzook der Materie", which is financially supported by the "Nederlandse Organisatie voor Zuiver Wetenschappelijk Onderzoek", for support.

\section{References}

[1] See, e.g.: G. Hutiray and J. Solyom, eds., CDW in Solids, Lecture Notes in Physics, vol. 217 (Springer, Berlin, 1985).

[2] H. Fukuyama and H. Takayama, in: Electronic Properties of Inorganic Quasi One Dimensional Materials, P. Monceau, ed. (Reidel, Dordrecht, 1985) p. 41.

[3] G. Kriza and G. Mihály, Phys. Rev. Lett. 56 (1986) 2529.

[4] S. Bhattacharya, M.J. Higgins, J.-P. Stokes and R.A. Klemm, Phys. Rev. B 38 (1988) 10093.

[5] R.M. Fleming and L.F. Schneemeyer, Phys. Rev. B 33 (1986) 2930.

[6] L. Pietronero and S. Strässler, Phys. Rev. B 28 (1983) 5863.

[7] L. Pietronero, in: Proc. Varenna School. 89th Course on Highlights of Condensed Matter Theory (North-Holland, Amsterdam, 1985) p. 442.

[8] P.B. Littlewood, Phys. Rev. B 33 (1986) 6694.

[9] S.N. Coppersmith and P.B. Littlewood, Phys. Rev. B 36 (1987) 311.

[10] C. Tang, K. Wiesenfeld, P. Bak, S. Coppersmith and P. Littlewood, Phys. Rev. Lett. 58 (1987) 1161.

[11] K. Kaneko, Prog. Theor. Phys. 74 (1985) 1033; Physica D 23 (1986) 436.

[12] K. Kaneko, Collapse of Tori and Genesis of Chaos in Dissipative Systems (World Scientific, Singapore, 1986).

[13] G. Gruener, A. Zawadowsky and P.M. Chaikin, Phys. Rev. Lett. 46 (1981) 511.

[14] P.B. Littlewood and R. Rammal, Phys. Rev. B 38 (1988) 2675.

[15] P.B. Littlewood and R. Rammal, Phys. Rev. Lett. 58 (1987) 524.

[16] Y. Pomeau and P. Manneville, Commun. Math. Phys. 74 (1980) 327.

[17] J.E. Hirsch, B.A. Huberman and D.J. Scalapino, Phys. Rev. A 25 (1982) 519.

[18] J.-P. Eckmann, L. Thomas and P. Wittwer, J. Phys. A 14 (1981) 3153.

[19] M. Versteeg, internal report University of Groningen (1986).

[20] D.S. Fisher, Phys. Rev. B 31 (1985) 1396.

[21] R. Heijungs, internal report University of Groningen (1987).

[22] P. Bak, C. Tang and K. Wiesenfeld, Phys. Rev. Lett. 59 (1987) 381; Phys. Rev. A 38 (1988) 364.

[23] S. Wolfram, Rev. Mod. Phys. 55 (1983) 601. 